\title{
Psychological Burden Associated with Lung Cancer Screening: A Systematic Review
}

\author{
Geena X. Wu, MD ${ }^{\mathrm{a}, 1}$, Dan J. Raz, MD ${ }^{\mathrm{a}}$, Laura Brown, MLS ${ }^{\mathrm{b}}$, and Virginia Sun, PhD, RN ${ }^{\mathrm{C}}$ \\ aDivision of Thoracic Surgery, Department of Surgery, and Lung Cancer Screening Program, City \\ of Hope, 1500 East Duarte Road, Duarte, California, 91010 \\ bLee Graff Medical and Scientific Library, City of Hope, 1500 East Duarte Road, Duarte, \\ California, 91010 \\ 'Division of Nursing Research \& Education, Department of Population Sciences, City of Hope, \\ 1500 East Duarte Road, Duarte, California, 91010
}

\section{Abstract}

Introduction-Lung cancer screening (LCS) with low dose computed tomography (LDCT) reduces mortality and is recommended for high-risk current and former smokers. Several potential harms associated with LCS have been identified, including the potential for psychological burden. To summarize the current state of the scientific knowledge on psychological burden associated with LCS, we performed a systematic search of the contemporary quantitative and qualitative research literature.

Methods-We included randomized controlled trials and cohort studies that evaluated the impact of LCS with LDCT on psychological burden and health-related quality of life (HRQOL) as assessed by validated and non-validated measures. PubMed, CINAHL, PsychINFO, and Scopus were searched for English language articles published between 2004 and January 2015. Data abstraction and quality assessment were conducted by two independent reviewers.

Results-Thirteen studies were included that met our inclusion criteria. Overall, results were variable with some studies reporting worse psychological burden for patients with indeterminate results at pre-screening, post-screening and short-term follow-up ( $<6$ months post-screen). These adverse effects diminished or resolved at long-term follow-up ( $>6$ months post-screen).

Conclusion-LCS may be associated with short-term adverse psychological burden, particularly after a false positive result. However, these adverse effects diminished over time. The current evidence is small, with limitations in study design and use of outcome measures. More highquality research is needed to determine the frequency, duration, and overall magnitude of LCSrelated psychological burden in non-clinical trial settings.

Correspondence: Virginia Sun, PhD, RN, Division of Nursing Research and Education, Department of Population Sciences, City of Hope, 1500 East Duarte Road, Duarte, CA 91010; Ph: 626/256-HOPE ext. 63122; fax: 626/301-8941; vsun@ coh.org.

${ }^{1}$ Present address: Department of Surgery, Maricopa Integrated Health System, 2601 East Roosevelt Street, Phoenix, AZ 85008

Publisher's Disclaimer: This is a PDF file of an unedited manuscript that has been accepted for publication. As a service to our customers we are providing this early version of the manuscript. The manuscript will undergo copyediting, typesetting, and review of the resulting proof before it is published in its final citable form. Please note that during the production process errors may be discovered which could affect the content, and all legal disclaimers that apply to the journal pertain. 


\section{Keywords}

Lung cancer screening; psychological distress; anxiety; health-related quality of life; shared decision-making

\section{Introduction}

Lung cancer is the leading cause of cancer mortality for both men and women in the United States and worldwide. ${ }^{1}$ An estimated 158,040 Americans will die from lung cancer in 2015, more than colon, breast, and pancreatic cancers combined. ${ }^{2}$ Compared to other leading cancer sites such as colon and breast, which have better outcomes due to successful screening, the 5-year survival rate for lung cancer is only $16.6 \% .^{3}$ In contrast, the ten-year survival of screen-detected lung cancers may be as high as $88 \% .{ }^{4}$ The National Lung Screening Trial (NLST) demonstrated that annual low-dose radiation computed tomography (LDCT) scans for three years improved lung cancer mortality by $20 \%$ when compared to chest $\mathrm{x}$-ray. ${ }^{5}$ This finding has led the US Preventative Services Task Force (USPSTF) and a number of professional medical organizations, including the Centers for Medicare and Medicaid Services (CMS), to support LCS with LDCT in high risk current and former smokers. ${ }^{6-8}$

One of the risks of LCS highlighted by most screening guidelines is the potential for psychological burden, such as anxiety related to false positive or, synonymously, indeterminate results. ${ }^{9}$ Screening may result in anxiety and psychological distress, which can potentially impact more multidimensional outcomes such as overall health-related quality of life (HRQOL). Psychological burden can potentially occur at various time points throughout the screening process (before screening, after screening while waiting for screen results, after a positive result, after a positive workup), and can vary by severity (mild to severe). ${ }^{10}$ Twenty-four percent of patients undergoing LDCT in NLST had pulmonary nodules identified, although $96 \%$ of these were benign. While advances in nodule management such as Lung Imaging Reporting and Data System (Lung-RADS) have minimized the number of positive studies to $10 \%$, the high rate of false positive exams suggests that many patients are at risk for screen-related distress. However, the magnitude, duration, frequency, and trajectory of LCS-related psychological burden is largely unclear. Therefore, we performed a systematic search of the contemporary research literature to summarize the current state of the scientific knowledge on psychological burden associated with LCS. We focused on gathering information on the number of published studies and select study characteristics, including follow-up period and findings. Special attention was given to describing the type of outcome measures used to assess psychological burden in each study. Finally, we classified study findings on the potential impact of LCS on psychological burden pre-screen (baseline) and post-screen (short-and long-term). 


\section{Materials and Methods}

\section{Search Strategy}

The Preferred Reporting Items for Systematic Reviews and Meta-Analyses guidelines (PRISMA) were followed for this review. ${ }^{11}$ We conducted a systematic literature search using PubMed, PsychINFO, CINAHL and Scopus electronic databases. In an effort to more efficiently identify relevant studies that reflect the most current practices in LCS with LDCT, we restricted our search to English language articles published in the last 10 years between January 2004 and January 2015. A research librarian with experience and expertise in cancer worked with the authors to develop a list of terms and Medical Subject Heading (MeSH) to further refine the search procedures. Key words included psychology, psychosocial, psychiatric, quality of life, distress, depression, anxiety, fear, risk perception, lung, early detection of cancer, cancer screening, and neoplasm (search strategies for the databases are available on request).

\section{Review Process}

Inclusion criteria for articles in the final full-text review included the following: 1) report results of an empirical study, 2) relate to LCS using LDCT, and 3) present findings on patient self-report of psychological burden and related outcomes (eg HRQOL, psychological distress, depression). We chose to include studies that utilized both validated and nonvalidated outcome measures of psychological burden to examine the impact of selected measures on study findings. All other studies that did not meet the criteria described were excluded, including case reports, commentaries, systematic reviews and meta-analyses. Two authors (GW, VS) independently reviewed all titles and abstracts from the initial search and reached agreement on whether selected abstracts should be retained. Where discrepancies occurred, the titles and abstracts were reviewed and discussed collectively until consensus was reached. Full text versions of the articles retained from the initial review were obtained. The full-text articles were reviewed by two authors (GW, VS) independently. The bibliography of each reviewed article was also perused for other relevant studies. Consensus was reached on articles that should be included in the final full text review.

\section{Data Abstraction}

Study characteristics from articles that met the inclusion criteria and selected through the two-stage review process were obtained. One author (VS) recorded the following details of each included study: year, location, patient population, sample size, study design, screening procedure and results, outcome measures, and key findings. A second author $(\mathrm{GW})$ reviewed all abstractions for verification, completeness, and accuracy. Any discrepancies between reviewers were resolved by further discussions until a consensus was reached. Selected articles were further classified based on timing of outcome assessment. Outcome measures recorded less than 6 months after initial screening were considered short-term impact of LCS whereas those taken 6 months or more after screening were considered long-term impact of LCS. 


\section{Results}

Figure 1 presents the flow diagram that outlines the approach for stepwise selection of articles included in the final full-text review. From an initial total of 2,113 articles, 13 studies that assessed the psychological burden of LCS and met the inclusion criteria were selected for review. Study characteristics of the 13 reviewed articles are summarized in Table 1 . The majority of studies were derived from three large randomized controlled trials evaluating the utility of LDCT for LCS. Five studies were based from Denmark, Belgium, and the Netherlands as part of the NELSON trial which compared LDCT with no screening in current or former smokers (quit less than 10 years) aged 50 to 75 who smoked more than 15 cigarettes/day for more than 25 years, or more than 10 cigarettes/day for more than 30 years. ${ }^{12}$ One study was based from the NLST which recruited participants between 55 and 74 years of age with at least a 30 pack-year smoking history and if a former smoker, had quit within 15 years, and randomized patients to LDCT or chest radiography. ${ }^{13}$ Another four studies were derived from the Dutch Lung Cancer Screening Trial (DLCST) which compared LDCT to no screening for 5 rounds and included current and former smokers (quit after age 50 and $<10$ years prior) aged 50-70 years with 20 or more pack-years. ${ }^{14}$

Table 1 presents study characteristics and findings from the articles included in the final full text review. Three articles included in our review were based on cohort studies evaluating LCS. One study was derived from the Pittsburg Lung Screening study (PLuSS) which included a cohort of 50-79 year-old current and former smokers (quit within 10 years) who smoked more than a half pack a day for more than 25 years and were screened with LDCT. Vierriko et al. evaluated the psychological burden of using spiral chest CT for LCS in 633 asbestos-exposed workers from Finland with variable smoking history. Lastly, 60 individuals with extensive family history of lung cancer (three blood relatives with lung cancer with at least one first degree relative) who underwent spiral CT for LCS were surveyed regarding their risk perception and concern for lung cancer before and after the process.

\section{Baseline (Pre-Screen) Psychological Burden}

Most studies measured baseline pre-screen anxiety and demonstrated varied results. With the exception of NLST HRQOL study participants who were more likely to be female, white, more educated, and unmarried compared to controls ${ }^{15}$, the screening groups of the remaining studies did not differ significantly in patient characteristics from control groups. ${ }^{16-20}$ Pre-screen assessments from the NELSON trial $(\mathrm{T} 0=$ pre-randomization; $\mathrm{T} 1=$ post-randomization screening group) did not demonstrate statistically significant differences among subjects who eventually received negative CT scan results and those who eventually received indeterminate results in all measures of HRQOL, general anxiety, and lung cancer specific distress. ${ }^{18}$ At T1, screening group subjects had HRQOL and general anxiety scores that were comparable to those of the Dutch general population. ${ }^{17}$ However, all respondents had certain HRQOL scores that were significantly worse after randomization than before ( $\mathrm{P}<0.05$ for EQ-5D, $\mathrm{P}<0.001$ for STAI 6 and IES).${ }^{18}$ In addition, a subset of patients who reported most discomfort while awaiting screening results had significantly worse lung cancer specific distress at randomization than those that found other aspects of CT scanning most discomforting. ${ }^{17}$ When baseline perceived risk was evaluated in the NELSON trial 
population, $14.6 \%(n=47 / 324)$ of participants reported high affective risk 1 day before screening and these participants had significantly worse measures of lung cancer-specific distress and general HRQOL than those in the low affective risk group $(\mathrm{p}<0.01) .{ }^{21}$ In a separate study on the same patient population, it was concluded that pre-screen informed decision-making, defined as adequate knowledge and a positive attitude toward LCS, had no effect on any measures of HRQOL except a better mental component score for those who made an informed decision $(\mathrm{p}=0.003) .{ }^{19}$ In the United States, NLST screened and control participants did not report significant differences in baseline HRQOL and anxiety measures. ${ }^{15}$

Rasmussen et al. reported that DLCST participants in the control group had significantly worse psychosocial consequences when compared to the screened group at baseline $(\mathrm{p}<0.022) .{ }^{22}$ Another study comparing DLCST participants to a sample of the general population at baseline found significantly higher negative psychosocial scores in the latter group in all psychological burden measures. This was attributed to significant differences between the trial participants and a comparable population control, including more women, higher socio-economic status, longer education, higher employment rate, and more central urban location in the trial group. ${ }^{23}$ In contrast, two separate DLCST-based studies evaluated trial participants and equivalent general population controls at baseline and found no statistically significant differences in the outcomes measures utilized, including consequences of screening $(\mathrm{COS})^{16}$ and use of anxiolytics or antidepressants. ${ }^{20}$

A study derived from the PLuSS demonstrated lower baseline anxiety and fear of cancer in individuals who were eventually found to have a suspicious screening result compared to those who eventually received an indeterminate or negative result. ${ }^{24}$ In a population at highrisk for lung cancer (based on family history), 64-74\% had high pre-screen lung cancer risk perception and $94 \%$ of participants reported thoughts and concerns about developing lung cancer that did not affect mood or interfere with daily activity. ${ }^{25}$ One third of asbestos workers undergoing LCS thought that they were at risk for lung cancer at baseline. Additionally, baseline anxiety was higher in the false positive group than in the negative group. ${ }^{26}$

\section{Short-Term (Immediate and <6 Months Post-Screen) Psychological Burden}

Short-term (immediate post-screen and <6 months) LCS-related psychological burden also varied by trial and screening results. The NLST did not demonstrate significant difference in HRQOL or anxiety among LCS participants that received false positive, significant incidental or negative results at 1 month after screening. However, patients who had true positives had lower scores on the mental component aspect of the SF-36 compared to baseline (OR 3.95 CI -5.87, $-2.04 \mathrm{p}<0.001$ ), which signified worse anxiety, as well as higher STAI ratio (OR $1.47 \mathrm{CI} 1.16-1.88, \mathrm{p}<0.01$ ), or worse HRQOL, than those with false positive, incidental, and negative results. ${ }^{15}$ The NELSON trial reported that $46.4 \%$ of participants reported discomfort and 50.5\% reported dread or fear while waiting for results, and this subgroup had significantly worse measures of HRQOL and lung cancer-specific distress as measured by STAI- 6 and IES, respectively ( $\mathrm{P}<0.01$ ). In addition, $76 \%$ of respondents reported the most discomfort while awaiting CT scan results, and had worse 
STAI-6 and IES scores immediately post-screen compared with those who reported most discomfort with other aspects of CT scanning ( $\mathrm{p}<0.05$ and $<0.01$, respectively). ${ }^{17}$ In a separate study from the same trial, there was no significant difference immediately postscreening in any measures of HRQOL or anxiety between the negative and indeterminate result groups. However at 2 months follow up, participants with indeterminate results had higher IES scores, indicating worse lung cancer specific distress than in the negative result group $(\mathrm{P}<0.01)$. In the negative result group, IES scores improved at two months compared to post-screen and to baseline scores ( $\mathrm{P}<0.01$ for both). In the indeterminate group, EQ-5D scores measuring HRQOL and IES scores worsened at 2 months compared to post-screen and baseline scores ( $\mathrm{P}<0.01$ for both). Likewise, STAI-6 scores suggested increased anxiety from baseline to 2 months in those with indeterminate results $(\mathrm{P}<0.05) .{ }^{18}$

Similar short-term findings were reported from the PLuSS cohort that demonstrated increased general anxiety measures with indeterminate or suspicious screening results immediately following and up to 1 month after screening. Fear of cancer scores (PCQ) increased after LDCT for screenees with suspicious results and were relatively unchanged for the negative or indeterminate groups. Perceived risk of cancer decreased in short-term follow up for those with negative screen results and increased for those with indeterminate or suspicious results. For all categories, perceived risk of cancer was higher than objective risk. However, perceived risk of cancer was accurately estimated to be high by those who had a suspicious screening result, while the negative and intermediate groups estimated much higher perceived risk than their actual objective risk. Additionally, higher education and married status were associated with lower anxiety while current smoking status was associated with higher anxiety. Current smokers and women experienced higher fear of cancer whereas the opposite was true for those with higher education. ${ }^{24}$ Similarly, for subjects undergoing screening for extensive family history of lung cancer, short-term evaluation at 1 month after screening demonstrated increased levels of worry, concern and perceived risk of cancer. ${ }^{25}$ In contrast, asbestos workers who received a negative or false positive LDCT screening result experienced decreased anxiety ( $\mathrm{p}<0.001)$ compared to baseline. There were also no differences in post-screening perceived risk of or worry for lung cancer among participants with negative or false positive results. ${ }^{26}$

\section{Long-Term (>6 Months Post-Screen) Psychological Burden}

Long-term adverse psychological burden of LCS was reduced or absent in most studies. At 6 months follow-up, only NLST HRQOL participants who received true positive results had worse HRQOL and anxiety measures from baseline. There was no difference in HRQOL or anxiety among the negative, false positive, or significant incidental finding groups from baseline to 6 months after screening. ${ }^{15}$

HRQOL and anxiety also did not change 6 months after baseline for participants in the NELSON trial and were not significantly different between the negative and indeterminate groups. ${ }^{17}$ Long-term assessment of lung cancer specific distress in low and high perceived risk groups showed that although IES scores were significantly lower at 6 months than at baseline for both groups, the high affective risk group had worse IES scores than the low affective risk group $(\mathrm{p}<0.01)$. However, there were significantly fewer subjects in the high 
affective risk group at 6 months than at pre-screen baseline. ${ }^{21}$ At 2 years, HRQOL and anxiety scores were not different between screen and control groups and any discrepancies in IES scores between indeterminate and negative result groups had resolved to baseline levels by the second round of screening. ${ }^{27}$ Long term follow up of PLuSS subjects at 6 months demonstrated that the short-term increase in anxiety in the indeterminate group had resolved and that the elevated perceived risk and fear of cancer experienced by those with suspicious screening results had diminished. ${ }^{24}$ By 6 months after screening, the same return to baseline of short-term increased worry, concern and perceived risk occurred in high risk screenees with extensive family history of lung cancer. ${ }^{25}$ In the screened population of asbestos workers, no significant long-term psychological differences were identified based on screening result. ${ }^{26}$

In assessing long-term psychosocial consequences of screening in DLCST participants, one study found significant increases in COS-LC scales in both control and screening groups 1 year after baseline CT. In addition, while no significant differences in COS-LC measures were found between the control and screen group at baseline, there were several significant differences in scores for anxiety, dejection, and self-blame at 1 year with the control group scoring worse than the screening group. This may be attributed to the significantly higher response rate of participants in the screening group (97\%) compared to that of the control group $(91.8 \%)$ in the prevalence screening round after randomization $(\mathrm{p}<0.0001)$, or to the fact that individuals with false positive results were excluded from analysis. ${ }^{16}$ Rasmussen et al. also reported significant increases in COS-LC scores for behavior, dejection, and poor sleep for both the screen and control groups after each annual screening up to 4 years. However, by years 3 and 4 (screening rounds 4 and 5), the scores for the screening group were closer to baseline. In general, the control group reported significantly worse psychosocial consequences compared to the screening group at all 5 rounds over 4 years. The control group also had more lung-cancer-specific negative psychosocial consequences compared to the screen group at 1, 2, and 4 years. ${ }^{22}$ Lastly, there was no difference in the use of anxiolytics or antidepressants after 3-year follow-up between the screen and control groups in this trial population. ${ }^{20}$

\section{Outcome Measures Utilized}

The selected articles utilized both validated and non-validated measures that were either general or condition-specific. Some studies used interviews to assess the effects of LCS on anxiety, fear, or worry of screening procedures, false positive results, and perceived risk. Outcome measures are summarized in Table 2 and include: the European Quality of Life (EQ-5D) with the visual analogue scale (VAS) for ranking self-impression of health; the two-component 36-item Short Form questionnaire (SF-36) with its shorter version the SF-12, both of which measure generic HRQOL (MCS = mental component summary and PCS = physical component summary); the State-Trait Anxiety Inventory (STAI-20 and STAI-6) with 20 and 6 questions, respectively, that measure generic anxiety; the impact of event scale (IES) which measures lung cancer specific distress; the consequences of screening (COS) and the lung-cancer-specific consequences of screening in Lung Cancer (COS-LC) which was developed and validated in the NELSON trial and the Dutch Lung Cancer Screening Trial (DLCST) and assesses the psychological burden of LCS ${ }^{22,28}$; the 
Psychological Consequences Questionnaire (PCQ) adapted from a validated measure in breast cancer screening to measure lung cancer fear.

\section{Discussion}

This systematic review identified and reviewed 13 studies that examined the impact of LCS on psychological burden. Ten of the studies were derived from three large randomized control trials evaluating the efficacy of LCS (NELSON, NLST, DLCST) while three studies reported psychological outcomes in smaller cohorts undergoing LCS (PLuSS, asbestos workers, individuals with lung cancer family history). Collectively, the current scientific evidence suggests that LCS has the potential to cause short-term psychological burden in individuals with an indeterminate scan result, although the adverse effects do not appear to persist long-term. This is in contrast to the current evidence in mammography screening for breast cancer, where indeterminate results requiring further investigation resulted in shortterm increased anxiety which persisted long-term for up to three years. ${ }^{29,30}$ Additionally, most of the reviewed studies did not report differences in psychological burden among individuals with false positive and true negative results. In some cases, a negative result led to decreased distress and anxiety while a true positive resulted in increased anxiety and worse HRQOL. ${ }^{15,26}$ Furthermore, individuals with indeterminate or suspicious results who endorsed high perceived risk of lung cancer or who experienced the most discomfort while waiting for CT screening results had increased anxiety, lung cancer-specific distress, and fear of lung cancer. ${ }^{17,21,24}$

There are several characteristics to the current body of evidence that warrants further discussion. First, our search yielded a small total number of studies evaluating psychological burden in LCS. This may perhaps be a result of the perception that psychological burden is trivial compared to the potential physical harms from screening, or the lack of a clear conceptual framework to guide high-quality studies on LCS-related psychological burden. Harris and colleagues recently proposed a framework to guide research examining the potential harms associated with LCS. The framework includes four key domains: physical harm, psychological harm, financial strain, and opportunity costs. ${ }^{31}$ Psychological harm, as proposed within this framework, can potentially occur at any step of the "screening cascade," but may be heightened at specific timepoints, such as receiving scan results or undergoing additional workup for a positive screen. ${ }^{31,32}$ Development and further refinement of conceptual frameworks on the psychological burden of LCS can potentially yield more high-quality evidence in future research.

The majority of studies selected in our review were conducted in the context of clinical trials that evaluated the effectiveness of LCS, comparing outcomes between screened versus nonscreened populations. Although these studies provide some evidence on LCS-related psychological burden, the findings may be biased because trial participation itself may have psychological effects. ${ }^{32}$ Furthermore, characteristics of trial participants are usually different from community nonparticipants; therefore the true magnitude of psychological burden may not be fully detected. Another important factor to consider is the context of the screening situation. Current recommendations are focused on screening high risk populations of current and former smokers. These individuals may already be experiencing feelings of 
guilt, shame, and anxiety based on factors such as perceived risk of developing lung cancer. ${ }^{31}$ Only six studies from our review included the assessment of these potential moderating factors.

Another important characteristic in our selected studies is the heterogeneity in outcome measures utilized to capture psychological burden. The majority of measures used were general rather than condition-specific. The use of general measures of psychological burden and HRQOL alone may not be as responsive to the subtle changes in psychological burden as condition-specific measures. ${ }^{10}$ There are few condition-specific measures in LCS with strong psychometric properties, with the exception of the COS-LC, which was used primarily in the European LCS trials. The addition of a more condition-specific measure may have resulted in differences in psychological burden outcomes in the European and US trials in our review. There is a need to develop and refine condition-specific psychological burden measures that are reliable and relevant to LCS populations.

The current Medicare coverage guidelines put forth by the Center for Medicare and Medicaid Services (CMS) includes a counseling and shared decision-making visit on the benefits and potential harms of LCS. Extensive counseling on LCS harms is a potentially promising strategy to decrease short-term psychological burden associated with LCS. Analysis of HRQOL and state anxiety data from the NLST study revealed no differences in these outcomes by screen-results (false-positive, true-positive, significant incidental findings), which the authors attributed partially to the extensive counseling that trial participants received. ${ }^{15}$ Quality counseling and shared decision-making can be guided by tools such as patient decision aids, which are designed to help individuals participate in complex decision-making related to health care options and to improve decision quality. ${ }^{33}$ Decision aids prepare patients to make informed, value-based decisions by providing quality information on the options available in easily understandable formats and coaching patients on communicating personal value to providers. ${ }^{33}$ As demand for LCS with LDCT increases, the provision of high quality information on the benefits and harms of screening is needed to promote informed decision-making, eliminate misperceptions, and reduce screen-related psychological burden.

Our review has some limitations. First, the small body of literature limits the ability to comment on the quality of data currently available. Second, our review focused only on psychological burden in the context of LCS; therefore, the findings may not be generalizable to other cancer screening settings. Finally, as previously discussed, our review yielded primarily studies that were conducted in an LCS clinical trial context; therefore, findings may be biased and not generalizable to non-participants.

In summary, based on our review, LCS did not appear to have substantial long-term impact on psychological burden, but potential short-term psychological burden was observed. More high-quality research conducted in non-clinical trial settings are needed to determine the frequency, duration, and overall magnitude of psychological burden associated with LCS. 


\section{References}

1. Centers for Disease Control and Prevention. CDC WONDER On-line Database. Vol Series 20. National Center for Health Statistics; 2013. No. 2P

2. Siegel RL, Miller KD, Jemal A. Cancer statistics, 2015. CA: a cancer journal for clinicians. Jan; 2015 65(1):5-29. [PubMed: 25559415]

3. Howlader, NNA., Krapcho, M., Garshell, J., Miller, D., Altekruse, SF., Kosary, CL., Yu, M., Ruhl, J., Tatalovich, Z., Mariotto, A., Lewis, DR., Chen, HS., Feuer, EJ., Cronin, KA. SEER Cancer Statistics Review, 1975-2011. National Cancer Institute; http://seer.cancer.gov/csr/1975_2011/

4. Henschke CI. International Early Lung Cancer Action Program I. Survival of patients with clinical stage I lung cancer diagnosed by computed tomography screening for lung cancer. Clinical cancer research : an official journal of the American Association for Cancer Research. Sep 1; 2007 13(17): 4949-4950. [PubMed: 17785541]

5. Aberle DR, Adams AM, et al. National Lung Screening Trial Research T. Reduced lung-cancer mortality with low-dose computed tomographic screening. The New England journal of medicine. Aug 4; 2011 365(5):395-409. [PubMed: 21714641]

6. Moyer VA. Force USPST. Screening for lung cancer: U.S. Preventive Services Task Force recommendation statement. Ann Intern Med. Mar 4; 2014 160(5):330-338. [PubMed: 24378917]

7. Wender R, Fontham ET, Barrera E Jr, et al. American Cancer Society lung cancer screening guidelines. CA Cancer J Clin. Mar-Apr;2013 63(2):107-117. [PubMed: 23315954]

8. Jensen, T., Chin, J., Ashby, L., Hermansen, J., Hutter, J. Final National Coverage Determination on Screening for Lung Cancer with Low Dose Computed Tomography (LDCT). The Centers for Medicare \& Medicaid Services (CMS); Feb 5. 2015 CAG-00439N

9. Bach PB, Mirkin JN, Oliver TK, et al. Benefits and harms of CT screening for lung cancer: a systematic review. JAMA : the journal of the American Medical Association. Jun 13; 2012 307(22): 2418-2429. [PubMed: 22610500]

10. DeFrank JT, Barclay C, Sheridan S, et al. The psychological harms of screening: the evidence we have versus the evidence we need. Journal of general internal medicine. Feb; 2015 30(2):242-248. [PubMed: 25150033]

11. Moher D, Liberati A, Tetzlaff J, Altman DG, Group P. Preferred reporting items for systematic reviews and meta-analyses: the PRISMA statement. PLoS medicine. Jul 21.2009 6(7):e1000097. [PubMed: 19621072]

12. van Iersel CA, de Koning HJ, Draisma G, et al. Risk-based selection from the general population in a screening trial: selection criteria, recruitment and power for the Dutch-Belgian randomised lung cancer multi-slice CT screening trial (NELSON). International journal of cancer. Journal international du cancer. Feb 15; 2007 120(4):868-874. [PubMed: 17131307]

13. Aberle DR, Adams AM, et al. National Lung Screening Trial Research T. Baseline characteristics of participants in the randomized national lung screening trial. J Natl Cancer Inst. Dec 1; 2010 102(23):1771-1779. [PubMed: 21119104]

14. Pedersen JH, Ashraf H, Dirksen A, et al. The Danish randomized lung cancer CT screening trial-overall design and results of the prevalence round. Journal of thoracic oncology : official publication of the International Association for the Study of Lung Cancer. May; 2009 4(5):608614.

15. Gareen IF, Duan F, Greco EM, et al. Impact of lung cancer screening results on participant healthrelated quality of life and state anxiety in the National Lung Screening Trial. Cancer. Jul 25.2014

16. Aggestrup LM, Hestbech MS, Siersma V, Pedersen JH, Brodersen J. Psychosocial consequences of allocation to lung cancer screening: a randomised controlled trial. BMJ open. 2012; 2(2):e000663.

17. van den Bergh KA, Essink-Bot ML, Bunge EM, et al. Impact of computed tomography screening for lung cancer on participants in a randomized controlled trial (NELSON trial). Cancer. Jul 15; 2008 113(2):396-404. [PubMed: 18484588]

18. van den Bergh KA, Essink-Bot ML, Borsboom GJ, et al. Short-term health-related quality of life consequences in a lung cancer CT screening trial (NELSON). Br J Cancer. Jan 5; 2010 102(1):2734. [PubMed: 19935789] 
19. van den Bergh KA, Essink-Bot ML, van Klaveren RJ, de Koning HJ. Informed decision making does not affect health-related quality of life in lung cancer screening (NELSON trial). Eur J Cancer. Dec; 2010 46(18):3300-3306. [PubMed: 20580546]

20. Kaerlev L, Iachina M, Pedersen JH, Green A, Norgard BM. CT-Screening for lung cancer does not increase the use of anxiolytic or antidepressant medication. BMC cancer. 2012; 12:188. [PubMed: 22621716]

21. Bunge EM, van den Bergh KA, Essink-Bot ML, van Klaveren RJ, de Koning HJ. High affective risk perception is associated with more lung cancer-specific distress in CT screening for lung cancer. Lung Cancer. Dec; 2008 62(3):385-390. [PubMed: 18468717]

22. Rasmussen JF, Siersma V, Pedersen JH, Brodersen J. Psychosocial consequences in the Danish randomised controlled lung cancer screening trial (DLCST). Lung cancer. Jan; 2015 87(1):65-72. [PubMed: 25433982]

23. Hestbech MS, Siersma V, Dirksen A, Pedersen JH, Brodersen J. Participation bias in a randomised trial of screening for lung cancer. Lung cancer. Sep; 2011 73(3):325-331. [PubMed: 21324544]

24. Byrne MM, Weissfeld J, Roberts MS. Anxiety, fear of cancer, and perceived risk of cancer following lung cancer screening. Medical decision making : an international journal of the Society for Medical Decision Making. Nov-Dec;2008 28(6):917-925. [PubMed: 18725404]

25. Sinicrope PS, Rabe KG, Brockman TA, et al. Perceptions of lung cancer risk and beliefs in screening accuracy of spiral computed tomography among high-risk lung cancer family members. Acad Radiol. Aug; 2010 17(8):1012-1025. [PubMed: 20599157]

26. Vierikko T, Kivisto S, Jarvenpaa R, et al. Psychological impact of computed tomography screening for lung cancer and occupational pulmonary disease among asbestos-exposed workers. European journal of cancer prevention : the official journal of the European Cancer Prevention Organisation. Jun; 2009 18(3):203-206.

27. van den Bergh KA, Essink-Bot ML, Borsboom GJ, Scholten ET, van Klaveren RJ, de Koning HJ. Long-term effects of lung cancer computed tomography screening on health-related quality of life: the NELSON trial. The European respiratory journal. Jul; 2011 38(1):154-161. [PubMed: 21148229]

28. Brodersen J, Thorsen H, Kreiner S. Consequences of screening in lung cancer: development and dimensionality of a questionnaire. Value in health : the journal of the International Society for Pharmacoeconomics and Outcomes Research. Aug; 2010 13(5):601-612. [PubMed: 20345552]

29. Brett J, Bankhead C, Henderson B, Watson E, Austoker J. The psychological impact of mammographic screening. A systematic review. Psychooncology. Nov; 2005 14(11):917-938. [PubMed: 15786514]

30. Brodersen J, Siersma VD. Long-term psychosocial consequences of false-positive screening mammography. Annals of family medicine. Mar-Apr;2013 11(2):106-115. [PubMed: 23508596]

31. Harris RP, Sheridan SL, Lewis CL, et al. The harms of screening: a proposed taxonomy and application to lung cancer screening. JAMA Intern Med. Feb 1; 2014 174(2):281-285. [PubMed: 24322781]

32. Harris RP. The Psychological Effects of Lung Cancer Screening on Heavy Smokers: Another Reason for Concern. JAMA Intern Med. Sep 1; 2015 175(9):1537-1538. [PubMed: 26214149]

33. International Patient Decision Aid Standards (IPDAS) Collaboration. [Accessed November 4, 2015] What are patient decision aids?. 2012. http://ipdas.ohri.ca/what.html 


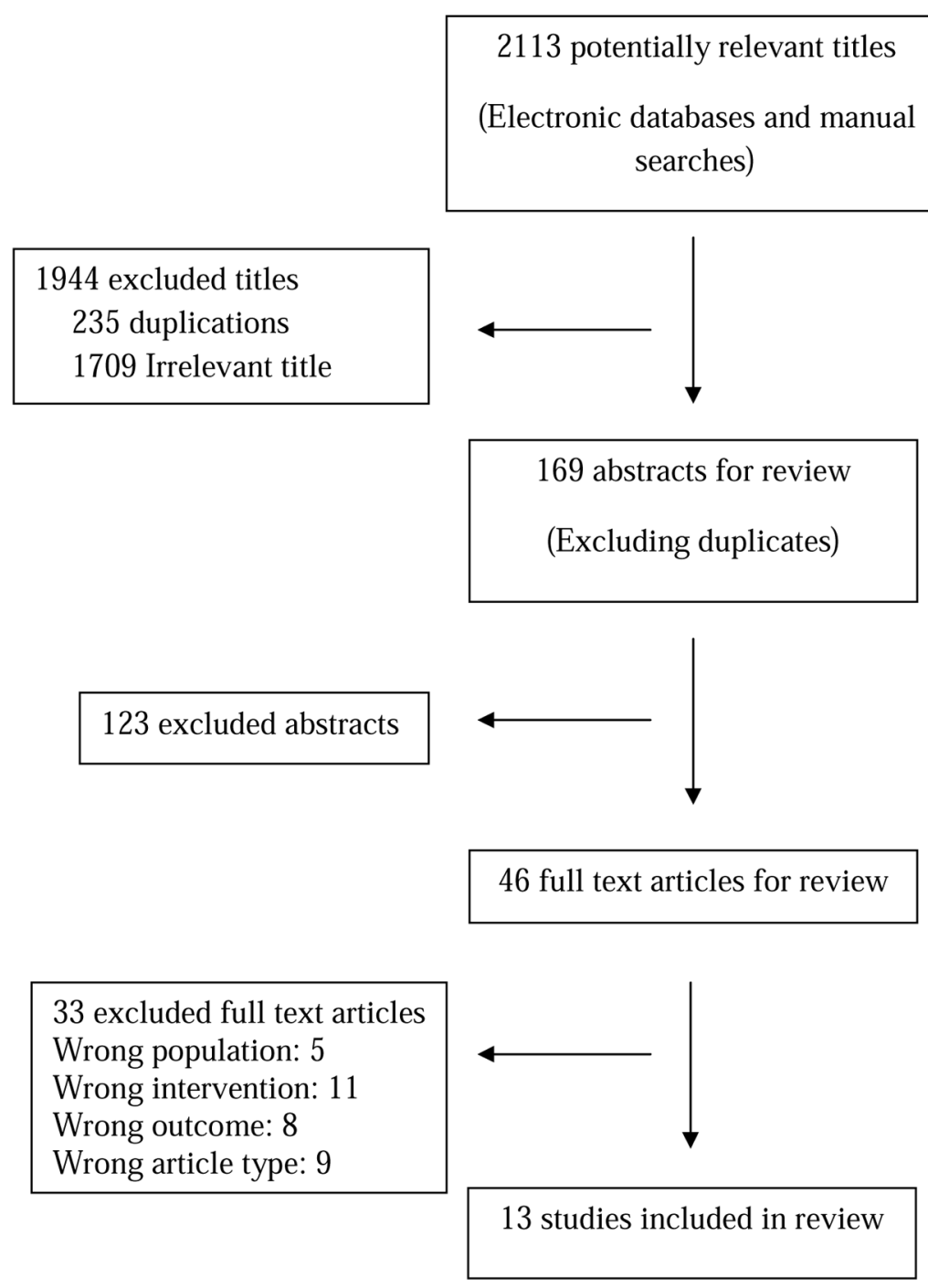

Figure 1.

Flow Diagram Representing Selection of Studies 


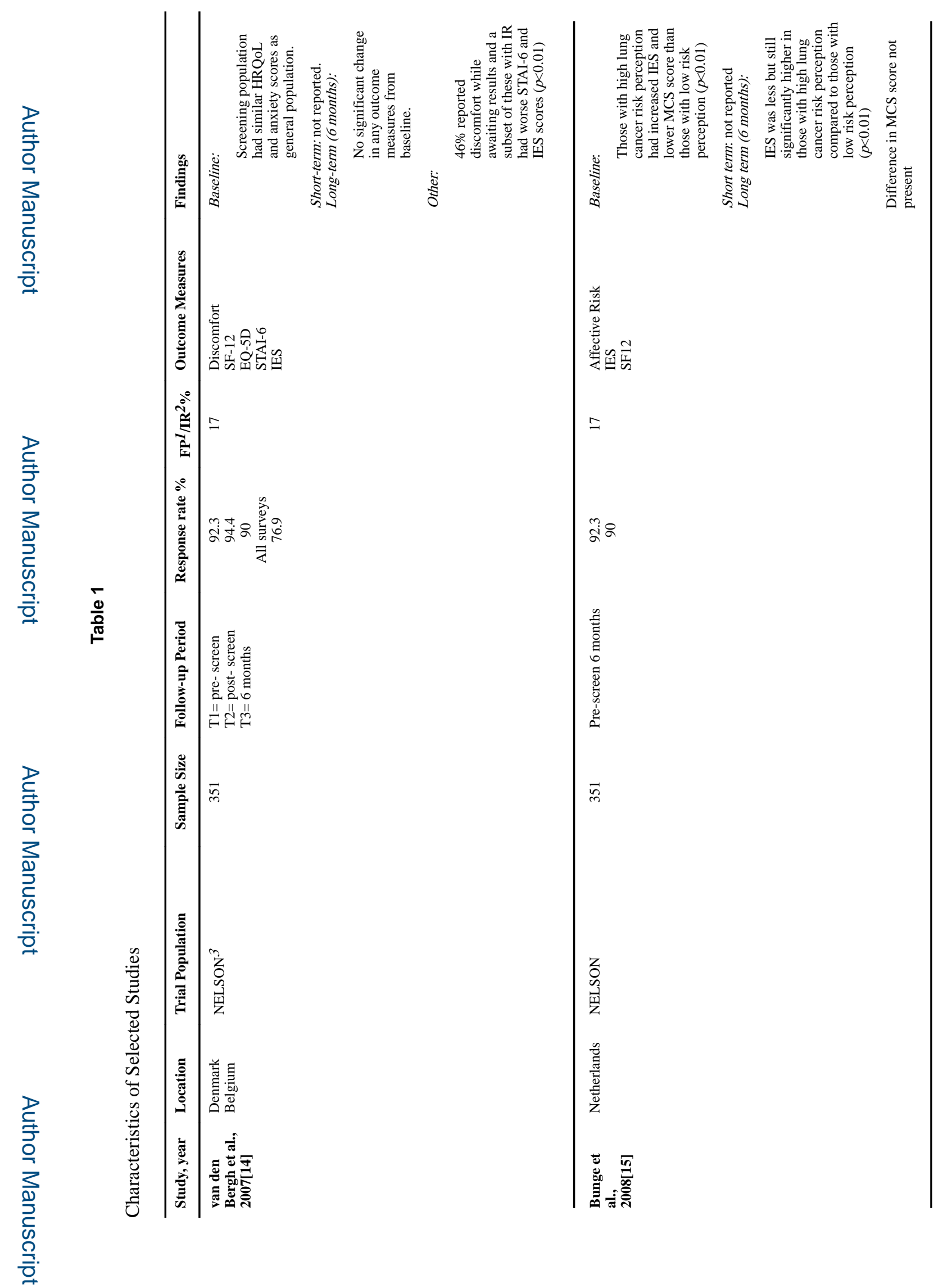

Clin Lung Cancer. Author manuscript; available in PMC 2017 September 20. 
Wu et al.

Page 14

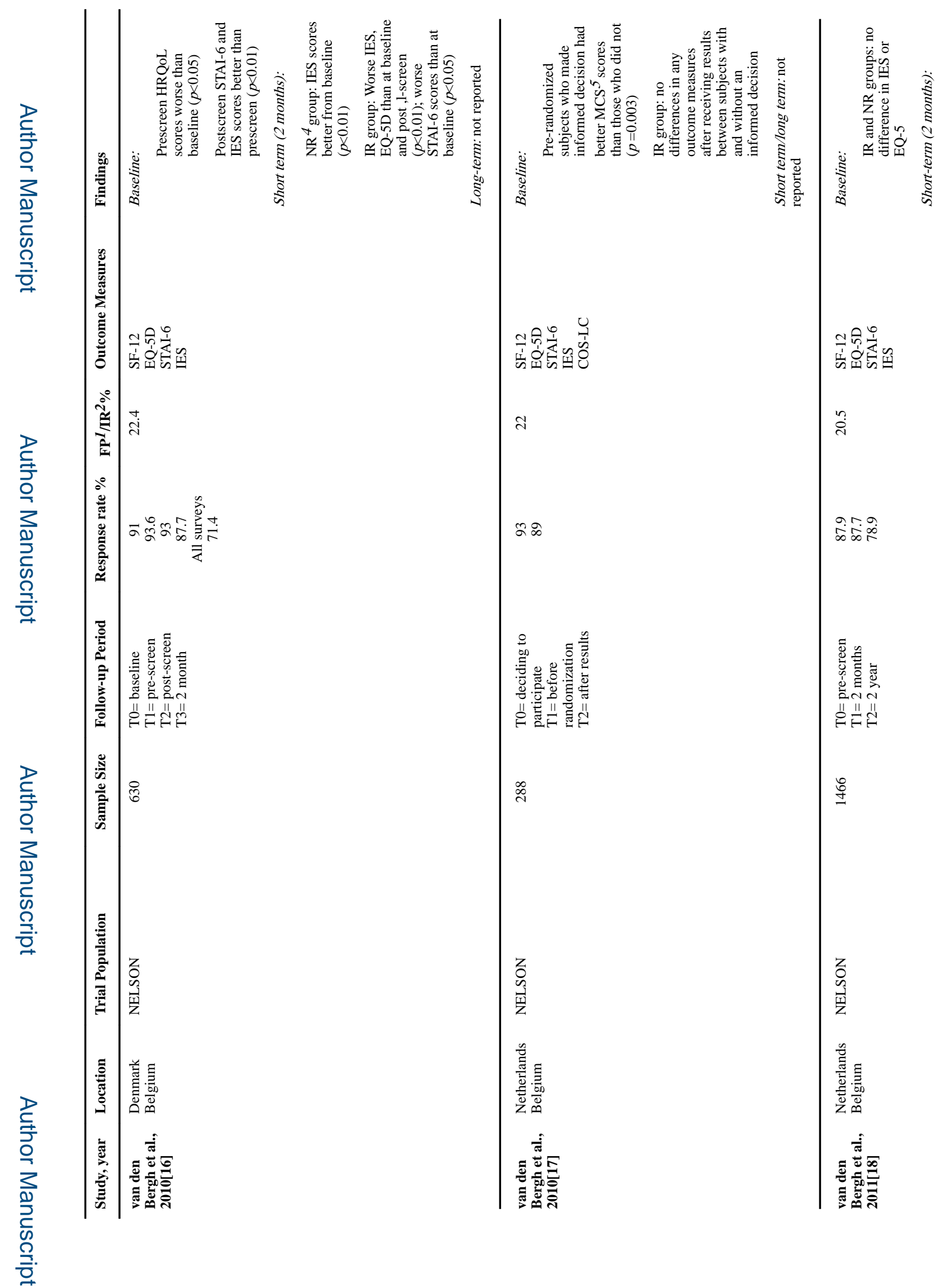

Clin Lung Cancer. Author manuscript; available in PMC 2017 September 20. 


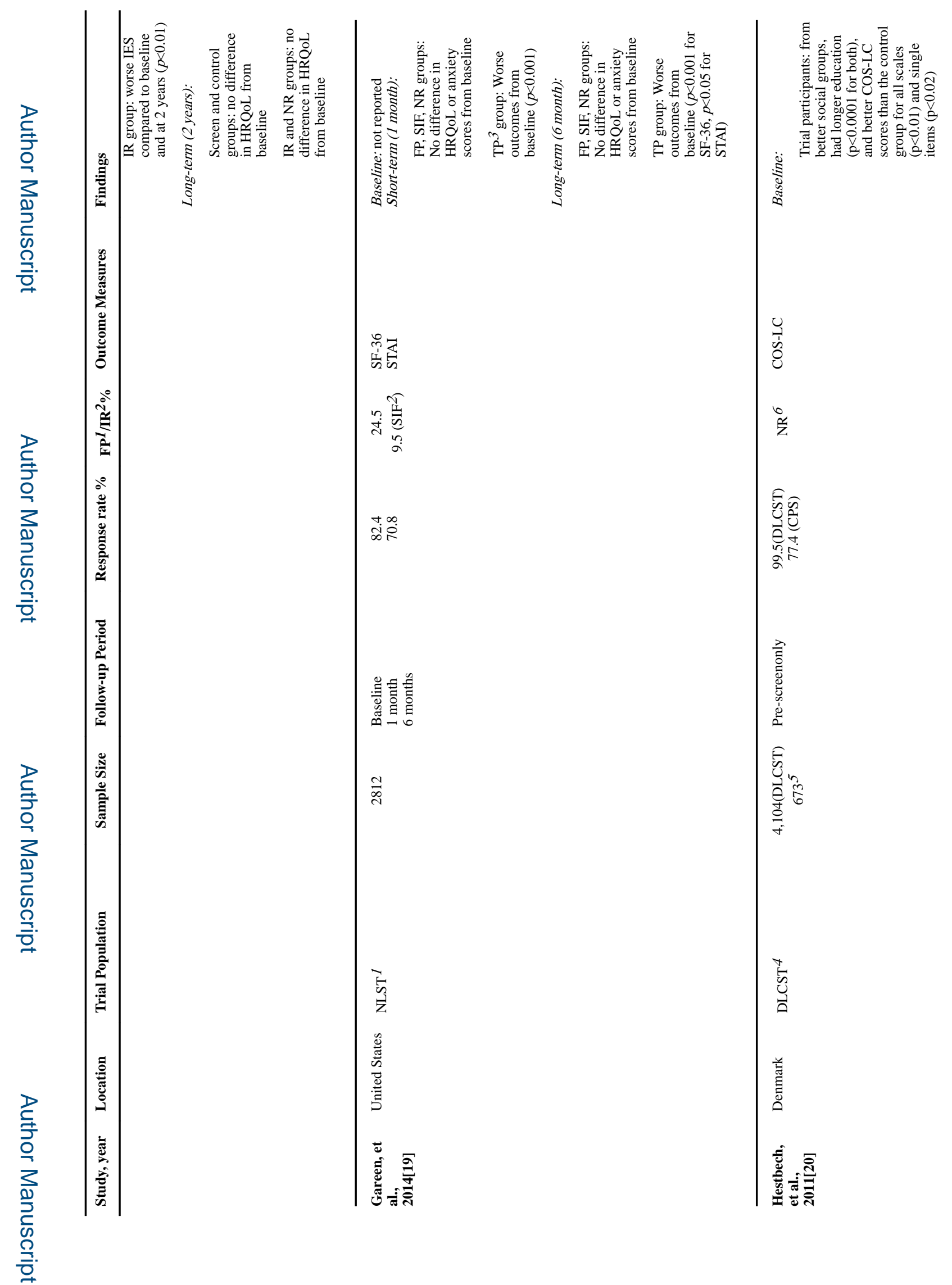

Clin Lung Cancer. Author manuscript; available in PMC 2017 September 20. 


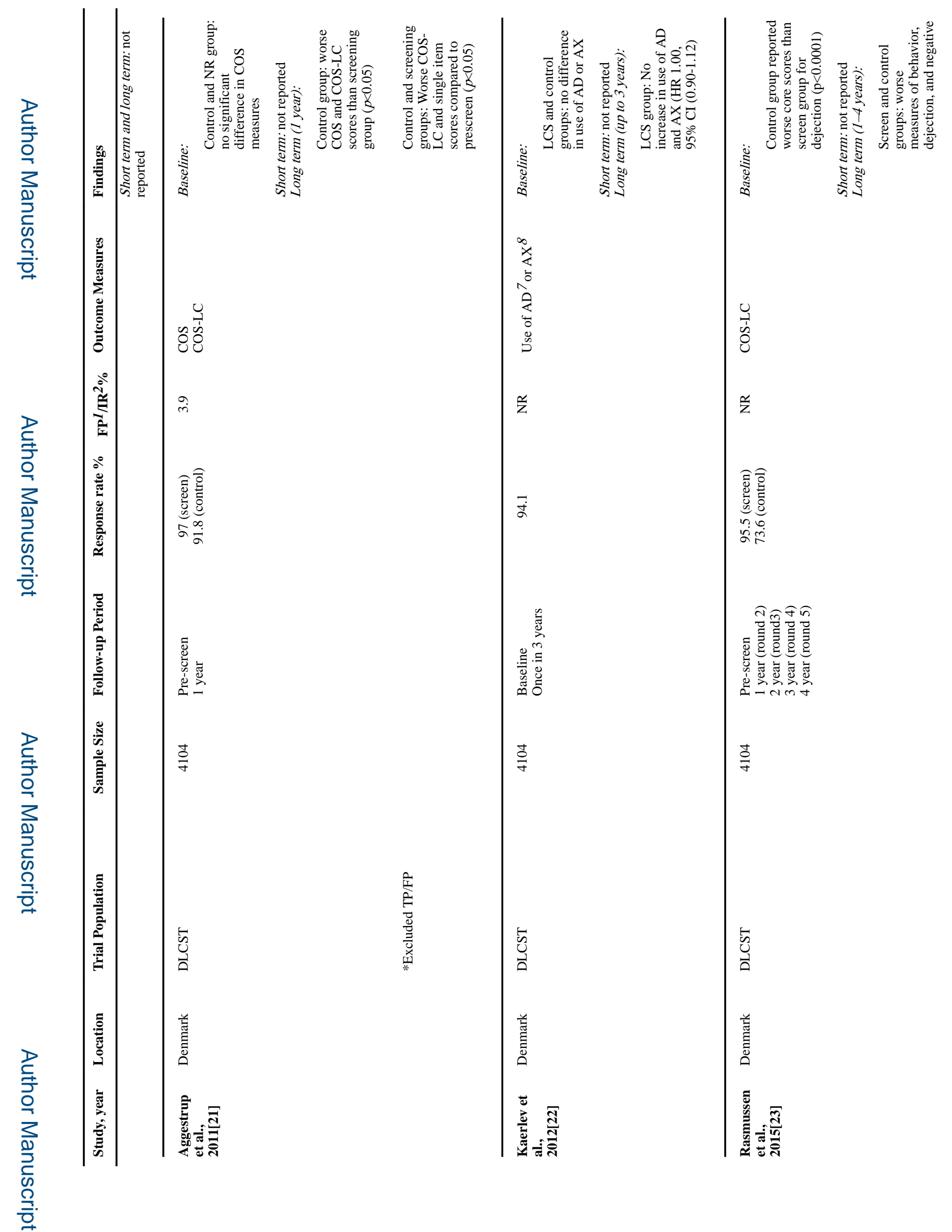

Clin Lung Cancer. Author manuscript; available in PMC 2017 September 20. 


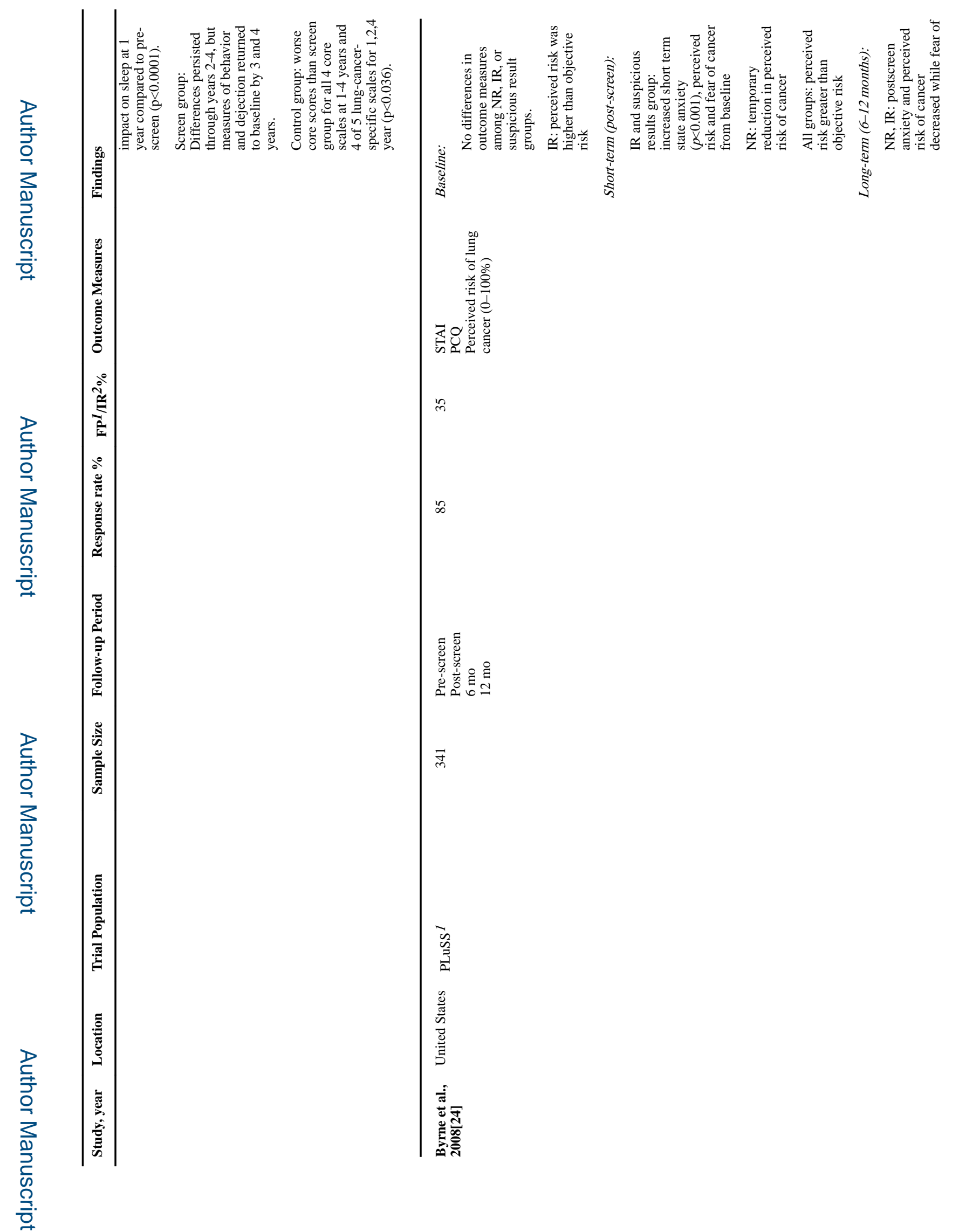


Wu et al.

Page 18

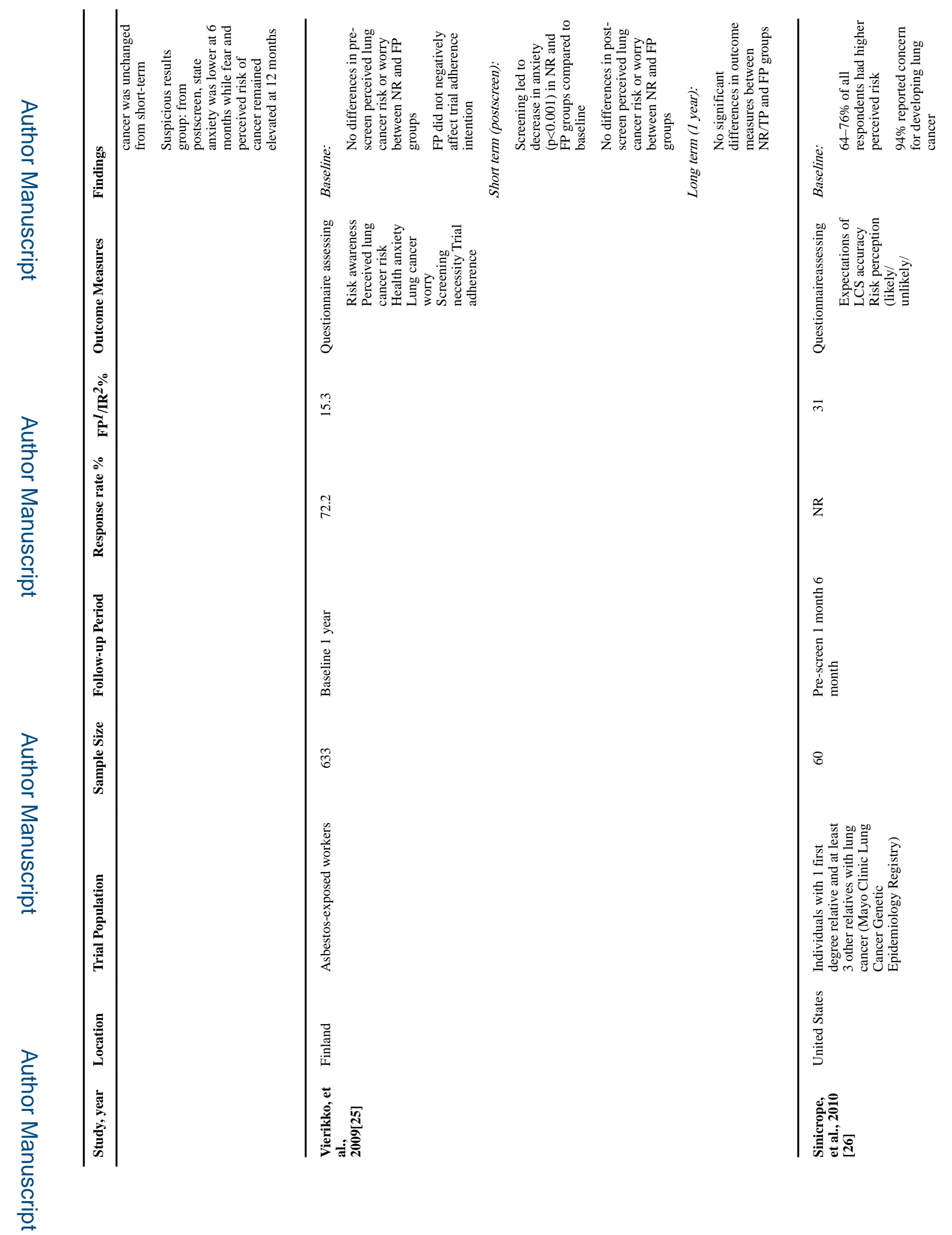

Clin Lung Cancer. Author manuscript; available in PMC 2017 September 20. 
Wu et al.

Page 19

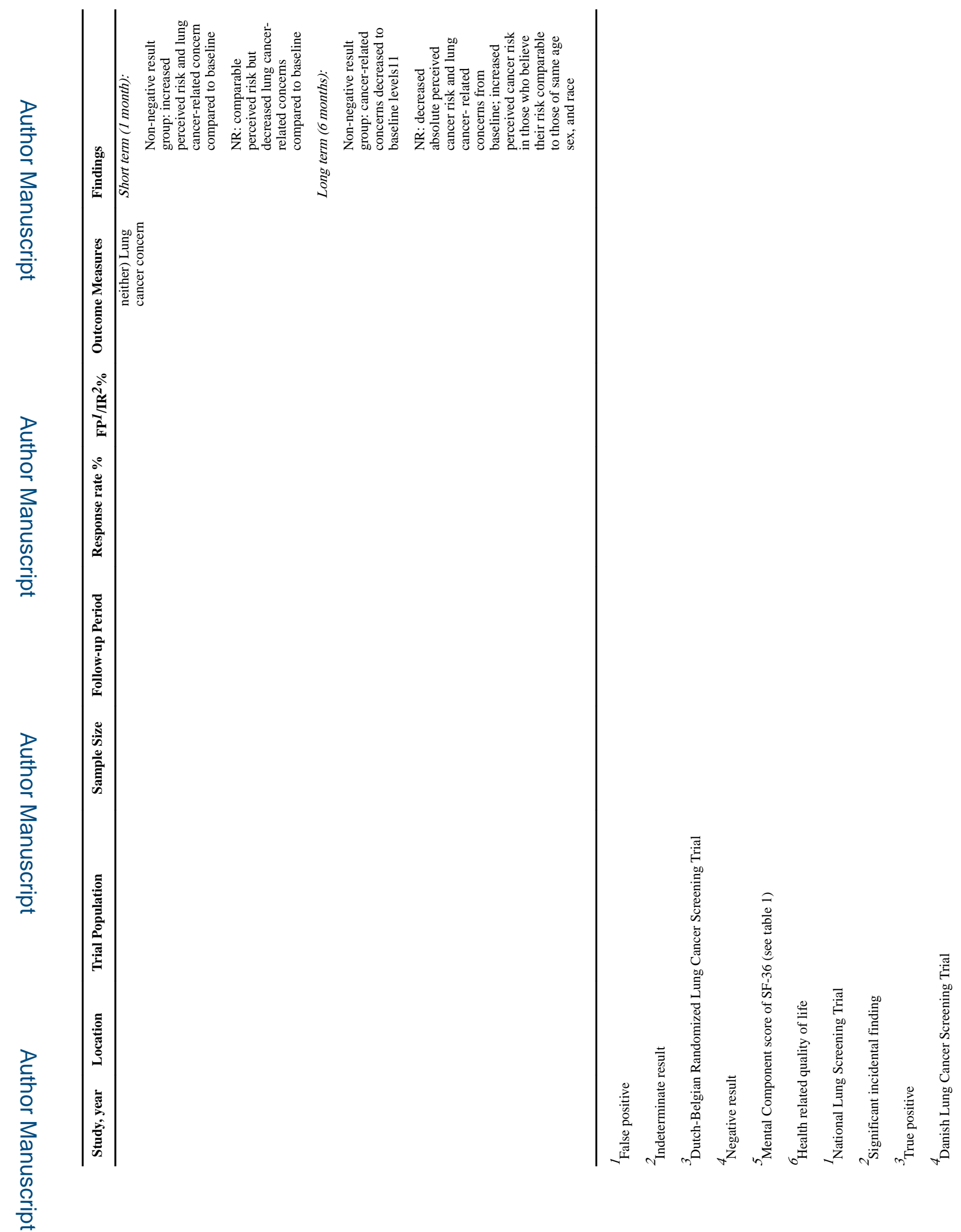

Clin Lung Cancer: Author manuscript; available in PMC 2017 September 20. 

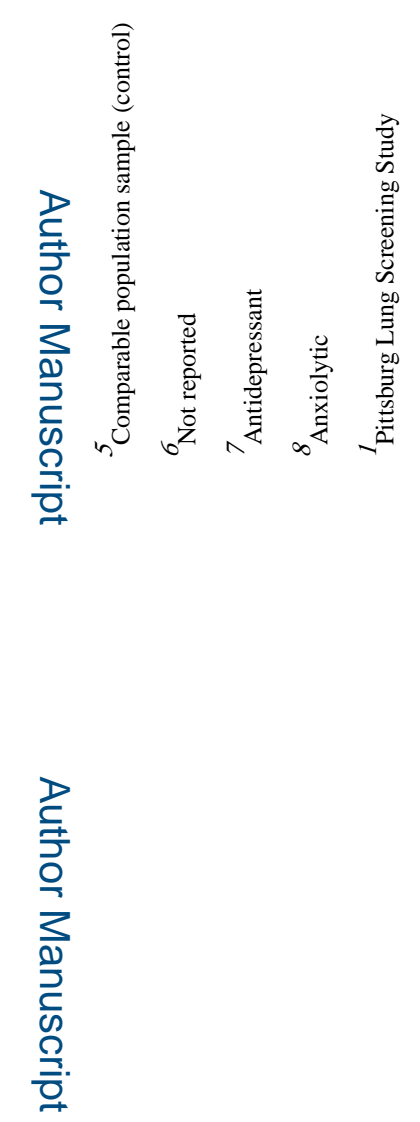

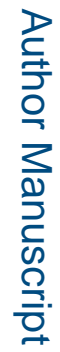

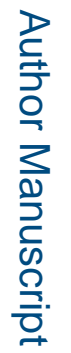

Clin Lung Cancer. Author manuscript; available in PMC 2017 September 20. 


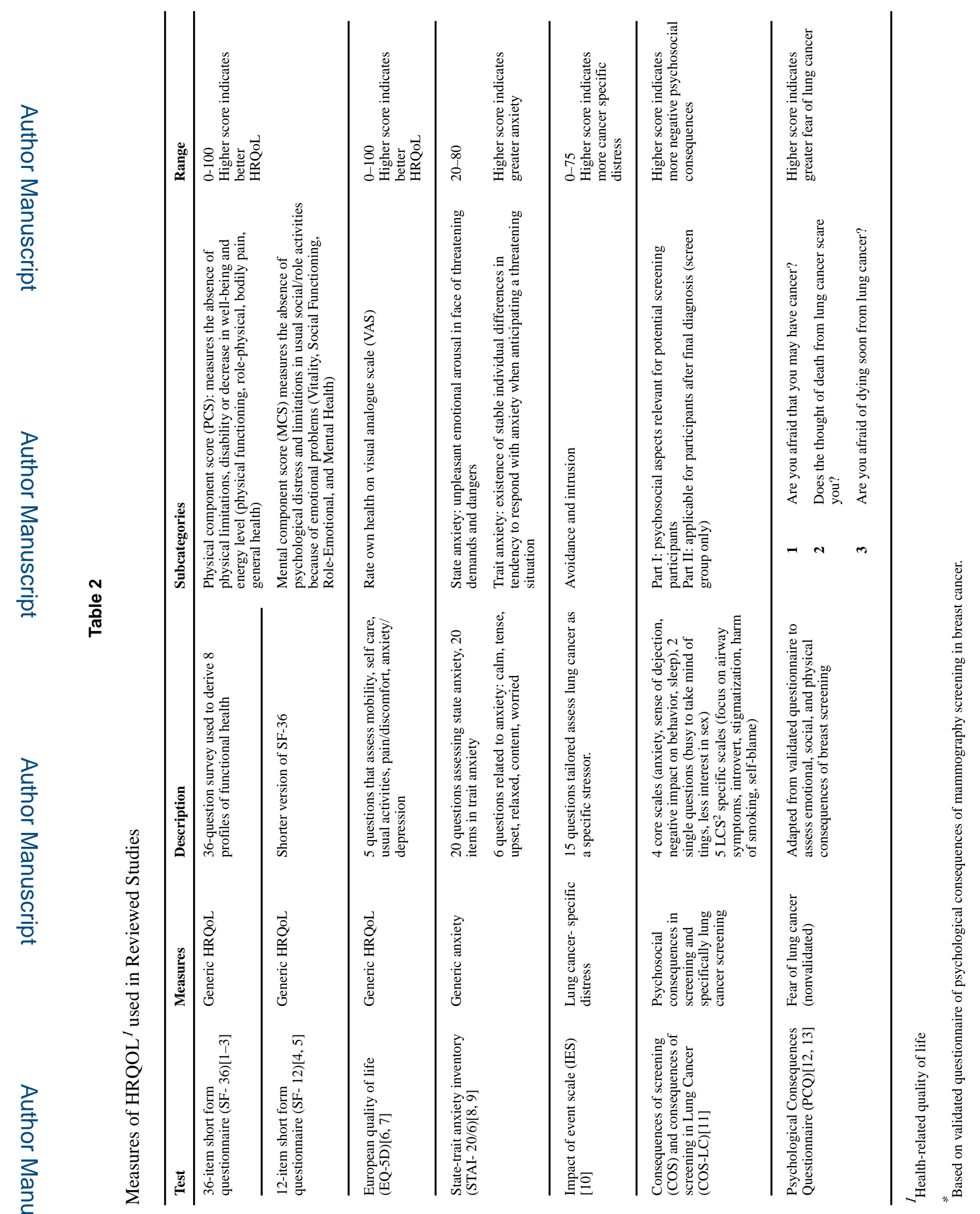

Clin Lung Cancer. Author manuscript; available in PMC 2017 September 20. 